\title{
Intraepidermal nerve fiber density and its application in sarcoidosis
}

M. Bakkers, MD

I.S.J. Merkies, MD, $\mathrm{PhD}$

G. Lauria, MD

G. Devigili, MD

P. Penza, MD

R. Lombardi, PhD

M.C.E. Hermans, MD

S.I. van Nes, MD

M. De Baets, MD, PhD

C.G. Faber, MD, PhD

Address correspondence and reprint requests to Dr. Mayienne Bakkers, Department of Neurology, Maastricht University Medical Centre, PO Box 5800, 6202 AZ, Maastricht, The

Netherlands

m.bakkers@mumc.n
Supplemental data at www.neurology.org

\section{ABSTRACT}

Background: Intraepidermal nerve fiber density (IENFD) is considered a good diagnostic tool for small fiber neuropathy (SFN).

Objectives: To assess stratified normative values for IENFD and determine the reliability and validity of IENFD in sarcoidosis.

Methods: IENFD was assessed in 188 healthy volunteers and 72 patients with sarcoidosis $(n=58$ with SFN symptoms, $n=14$ without SFN symptoms). Healthy controls were stratified (for age and sex), resulting in 6 age groups (20-29,30-39, .. up to $\geq 70$ years) containing at least 15 men and 15 women. A skin biopsy was taken in each participant $10 \mathrm{~cm}$ above the lateral malleolus and analyzed in accordance with the international guidelines using bright-field microscopy. Interobserver/intraobserver reliability of IENFD was examined. In the patients, a symptoms inventory questionnaire (SIQ; assessing SFN symptoms) and the Vickrey Peripheral Neuropathy Quality-of-Life Instrument-97 (PNQoL-97) were assessed to examine the discriminative ability of normative IENFD values.

Results: There was a significant age-dependent decrease of IENFD values in healthy controls, with lower densities in men compared with women. Good interobserver/intraobserver reliability scores were obtained ( $\kappa$ values $\geq 0.90$ ). A total of 21 patients with sarcoidosis had a reduced IENFD score (<5th percentile; 19 [32.8\%] in patients with SFN symptoms, 2 [14.3\%] in patients without SFN symptoms). The validity of the normative IENFD values was demonstrated by distinguishing between the SIQ scores and various PNQoL-97 values for the different patient groups.

Conclusion: This study provides clinically applicable distal intraepidermal nerve fiber density normative values, showing age- and sex-related differences. Neurology ${ }^{\circledR}$ 2009;73:1142-1148

\section{GLOSSARY}

IENFD = intraepidermal nerve fiber density; MUMC = Maastricht University Medical Center; NS = not significant; PNQoL$\mathbf{9 7}$ = Vickrey Peripheral Neuropathy Quality-of-Life Instrument-97; SFN = small fiber neuropathy; SIQ = symptoms inventory questionnaire.

Investigating sensory symptoms can be a diagnostic challenge. When a neuropathy is considered, neurologic examination and nerve conduction studies generally do not reveal abnormalities if predominantly small fibers are affected. ${ }^{1}$ There is no gold standard for diagnosing small fiber neuropathy (SFN), though a sensory neuropathy may present with small fiber involvement or indicate a disease causing pure small fiber dysfunction. ${ }^{2}$ Typically, patients with SFN report burning pain in the extremities, combined with autonomic dysfunction, such as dry eyes, changes in sweating, and gastrointestinal and urogenital dysfunction. ${ }^{3}$

Quantitative sensory testing such as temperature detection thresholds, sudomotor axon reflex, and cardiovascular autonomic testing are available techniques to determine small nerve fiber function. However, some tests have disadvantages, such as subjectivity, high burden for

\footnotetext{
From the Department of Neurology (M.B., I.S.J.M., M.C.E.H., C.G.F.), Maastricht University Medical Center, The Netherlands; Department of Neurology (I.S.J.M.), Spaarne Hospital, The Netherlands; Department of Neurology (I.S.J.M., S.I.v.N.), Erasmus Medical Center Rotterdam, The Netherlands; Neuromuscular Diseases Unit (G.L., P.P., R.L.), IRCCS Foundation, "Carlo Besta" National Neurological Institute, Milan, Italy; Neurologic Clinic (G.D.), University of Ferrara, Italy; and School for Mental Health and Neuroscience (M.D.B.), Maastricht, The Netherlands. Supported by a grant from the "Profileringsfonds" of the Maastricht University Medical Center, The Netherlands (project number 062053). This fund has had no role in the design and conduct of the study, nor in the collection, management, analysis, or interpretation of the data, in preparation, review, or approval of the manuscript.

Disclosure: Author disclosures are provided at the end of the article.
} 
patients, or low sensitivity and specificity. ${ }^{4}$ In SFN, loss of intraepidermal nerves is considered an objective diagnostic feature. ${ }^{5}$

There are several potentially treatable illnesses that may cause SFN. Diabetes mellitus is the most frequent underlying disease, but several other metabolic, inflammatory, and hereditary disorders cause SFN. ${ }^{2}$ Recently, SFN was demonstrated to occur in sarcoidosis. ${ }^{6}$ Only a small sample of patients with sarcoidosis has been examined so far.

Determination of intraepidermal nerve fiber density (IENFD) is considered a reliable technique to assess loss and regeneration of small nerve fibers. Several techniques have been adopted; the most commonly used are bright field immunohistochemistry and indirect immunofluorescence on punch biopsies. ${ }^{7-9}$ Reported interobserver/intraobserver variability is good. ${ }^{10}$

The aims of the current study are to present normative IENFD values, stratified for age and sex, for a collaborative group (Maastricht, The Netherlands; Milan and Ferrara, Italy), and to examine the interobserver/intraobserver reliability and discriminatory validity of IENFD in a cohort of patients with sarcoidosis.

METHODS Participants. Healthy controls. Healthy controls were recruited from hospital personnel, from relatives and friends of patients, at sports accommodations, and at informal meetings for the elderly, in The Netherlands and Italy. Healthy individuals were stratified for age and sex, forming 6 age groups $(20-29,30-39,40-49, \ldots$ up to 70 years and older) consisting of at least 15 men and 15 women each. Eligibility criteria were lucid consciousness, no neurologic symptoms, no history of alcohol misuse (defined as $>4$ IU/day), no use of drugs or history of diseases that may cause sensory deficit (e.g., chemotherapeutic agents in the past, malignancy, diabetes mellitus, thyroid dysfunction, renal failure, peripheral vascular disease, skin disorders), and normal findings at neurologic examination.

Patients. From October 2006 to June 2008, patients referred to the Maastricht Sarcoidosis Management Center, a referral center for sarcoidosis in The Netherlands, were screened for eligibility. Inclusion criteria for the study were age older than 18 years, no alcohol abuse, no use of immunosuppressant or neuropathy-causing drugs, no concomitant disease possibly causing SFN (normal laboratory findings for glucose, thyroid function, blood count, and renal and liver function tests), and a neurologic examination without signs of large fiber neuropathy or CNS involvement. Furthermore, nerve conduction findings (assessing median, peroneal, and tibial motor nerves, and median and sural sensory nerves under predefined conditions) had to be normal. All patients were diagnosed as having sarcoidosis in accordance with postulated diagnostic guidelines. ${ }^{11}$ We only recruited therapy-naive patients or patients who had not received immuno- suppressive therapy in the 6 months before inclusion, because the effect of these medications on small nerve fibers is unknown.

Assessments. Skin biopsy. Published European guidelines on the use of skin biopsy in the diagnosis of SFN were used.7 A 3 -mm punch biopsy was performed under local anesthesia (1\% lidocaine) $10 \mathrm{~cm}$ above the right lateral malleolus in all subjects. All biopsies were fixed in $2 \%$ paraformaldehyde-lysinesodiummetaperiodate and stored at $-80^{\circ} \mathrm{C}$ in a cryoprotective solution (20\% glycerol) before further processing. After cutting (Microm, HM560 Cryostar, Walldorf, Germany), the 50- $\mu \mathrm{m}$ thick sections were immunostained using a free-floating method: after bleaching, the sections were blocked with goat serum, incubated with the panaxonal marker PGP 9.5 (Ultraclone, Wellow, Isle of Wight, UK), and secondary antibody (biotinylated antirabbit immunoglobulin G, Biozol, Eching, Germany). The reaction was then visualized after endogenous peroxidase blocking, with the Vectastain ABC kit and Vector SG substrate kit (Vector Laboratories, Burlingame, CA). Epidermal length was measured and sections were counted with bright field microscopy on a stereology workstation (Olympus BX50 with PlanApo objective 40× [oil; $\mathrm{NA}=1.0]$, Tokyo, Japan, and stereology software StereoInvestigator version 7.00.3, Micro-BrightField, Williston, VT), applying the established counting rules. ${ }^{12}$ In brief, the rules state that to be counted, intraepidermal nerves have to cross or originate at the dermal-epidermal junction, and secondary branches and fragments are not counted. After fixing the specimens, at least 3 sections were analyzed under the light microscope. IENFD is reported as the mean IENF of these 3 sections per millimeter.

SFN symptoms inventory questionnaire. A simple SFN symptoms inventory questionnaire (SIQ) was constructed, based on international guidelines. ${ }^{13,14}$ The SIQ includes 12 inquiries addressing the following aspects: presence of palpitations, flushes, constipation or diarrhea, urination problems (incontinence or hesitation), changes in sweating pattern, restless legs, orthostatic dizziness, dry eyes or mouth, oversensitivity and sheet intolerance of the legs. Items focused on the presence or absence of these symptoms $(0=$ no symptoms to 12 = maximum number of symptoms).

Vickrey Peripheral Neuropathy Quality-of-Life Instrument-97. The Vickrey Peripheral Neuropathy Qualityof-Life Instrument-97 (PNQoL-97) has the Short-Form 36Item Health Survey as a generic core, supplemented with specifically neuropathy related questions, resulting in a 97-item scale, with 91 items distributed among 16 multiscale domains and 6 separately scored questions. ${ }^{15,16}$ The domains are physical functioning (11 items), role limitations due to physical health problems (6 items), pain (7), energy/fatigue (5), upper extremities (6), balance (8), self-esteem (6), emotional well-being (7), stigma (3), cognitive function (3), role limitations due to emotional problems (3), general health perceptions (7), sleep (5), social functioning (9), sexual function (2), and health distress (3). ${ }^{16}$ Each domain has a scoring range from 0 to 100 . A high score indicates better health. For the purpose of the current study, the original PNQoL-97 was translated-back-translated by 2 independent translators into Dutch in accordance with international guidelines. ${ }^{14}$ Ambiguous items were discussed until uniformity was obtained on all items. The Vickrey PNQoL-97 physical and mental component summary score (V-PCS, V-MCS) values were calculated. ${ }^{16-18}$

Standard protocol approvals, registration, and patient consent. The study was approved by the medical ethical committees of all participating centers and the CCMO (Central Committee for Human Related Research, identifier number p06.0066L/MEC 05-224). Written informed consent was obtained from all participants before inclusion. 
Study design. General aspects. Examination occurred in a comfortable, temperature-controlled room. All participants underwent a standardized interview, neurologic examination, and skin biopsy by a skilled investigator (M.B.). All selected patients were recruited at the department of neurology of the Maastricht University Medical Center (MUMC), The Netherlands. A patient was classified as having SFN symptoms when he or she reported at least 1 of the following symptoms, not otherwise explained: burning pain in extremities, dry mouth or eyes, changes in sweating, flushes, gastrointestinal dysfunction (constipation, diarrhea), cardiac symptoms (palpitation, dizziness on standing up), and urogenital dysfunction (sexual dysfunction, incontinence). Those without any of these symptoms were classified as having no SFN symptoms (patient control group). All patients completed the SIQ and PNQoL-97.

Reliability studies. All biopsies were coded, thus blinding the examiners. The IENFD of all participants at the MUMC was counted by 1 researcher (M.B.). Fifty randomly selected slides (25 patients plus 25 healthy controls) were also analyzed by a second examiner (C.G.F.) (local IENFD interobserver reliability). These 50 slides were recounted by the examiners (interval at least 2 weeks) for the IENFD intraobserver reliability scores of both examiners, without having access to the earlier findings. To determine interobserver reliability between laboratories, examiners at Milan (R.L.), Ferrara (G.D.), and Maastricht (M.B.) each counted 30 arbitrarily selected slides (healthy controls; 10 slides per center, circulating between institutes).

The SIQ was completed twice (interval: $2-4$ weeks) to examine its test-retest reliability.

Validity studies. The validity of the IENFD normative values was examined through discriminatory studies with the SIQ and PNQoL-97 domains and component sum scores in the patient group. In particular, we examined whether the normative data would help to discriminate between the obtained scores in these outcome measures that are based on patients' own perception (sarcoidosis patients without SFN symptoms vs those patients with SFN symptoms but normal IENFD scores vs those with SFN symptoms and abnormal IENFD).

Statistical analysis. Data handling and normative values. Data collection, entry, and management were performed using the Teleform automated processing system, striving to reduce the number of manual data entry errors. ${ }^{19}$ All analyses were performed using Stata 10.0 for Windows XP (StataCorp LP, College Station, TX). A value of $p<0.05$ was considered significant.

Normative IENFD reference values were calculated in healthy controls ( 5 th percentile values, corresponding to a chosen specificity of 95\%), depending primarily on age and sex. Quantile regression analyses were performed to obtain the normative data. A patient's IENFD was considered to be abnormal if the corresponding value was below the normative data 5th percentile lower limit, because this cutoff value has previously been used. ${ }^{20}$ Weight and height were analyzed for possible correlating confounding impact on IENFD normative values.

Reliability studies. Interobserver and intraobserver reliability scores for the IENFD and SIQ test-retest reliability were determined using weighted $\kappa$ statistic measures. ${ }^{21}$ The weights of the $\kappa$ were defined as $1-[(i-j) /(k-1)]^{2}(i=$ rows and $j=$ columns of the ratings by 2 observers or by the patients twice in time, $k=$ maximum number of possible ratings).

Validity studies. The discriminative capacity of the obtained IENFD normative values was investigated in 3 subgroups of patients (subgroup A: sarcoidosis patients without SFN symp- toms; subgroup B: sarcoidosis patients with SFN symptoms but normal IENFD values; subgroup C: sarcoidosis patients with SFN symptoms and impaired IENFD scores $[<5$ th percentile lower limit]) by comparing the obtained scores for SIQ plus PNQoL-97 values in the patient subgroups (analysis of variance with corrections according to Bonferroni multiple comparison tests for continuous variables; $\chi^{2}$ test for ordinal variables).

RESULTS General aspects. Recruitment was performed between January 2006 and June 2008. Skin biopsies were collected in 188 healthy volunteers (Maastricht $\mathrm{n}=105$, Milan $\mathrm{n}=44$, Ferrara $\mathrm{n}=$ 39), stratifying for age decade and sex (15 men and 15 women per age decade, range 20-82 years and older, forming 6 age decades). Most volunteers were white $(97 \%)$.

Between October 2006 and June 2008, 211 patients with sarcoidosis were referred to the MUMC (figure e-1 on the Neurology ${ }^{\circledR}$ Web site at www. neurology.org). Reasons for exclusion were immunosuppressive treatment (65), comorbidity that could cause neuropathy (30), CNS involvement (11) and large nerve fiber involvement found at neurologic or nerve conduction studies (21), and inability or unwillingness to attend examination (9). Eventually, 72 patients met the inclusion criteria and declared willingness to participate. Of these 72 patients, 58 were categorized as having symptoms not otherwise explained and possibly caused by SFN, and 14 were categorized as not having any SFN-related symptoms. All patients mentioned fatigue as one of their main problems. The participants' characteristics are presented in table 1 . No complications were encountered in either healthy volunteers or patients due to the skin biopsy.

Normative values. Normative IENFD reference values were calculated in 188 healthy controls. There was a significant age-dependent decrease of IENFD values, with lower scores for men compared with women (figure 1). Height and weight did not have any significant impact on the IENFD scores. The obtained normative scores were subsequently translated per age span and sex for clinical use, using the 5 th percentile as a cutoff value (table 2 ).

Reliability and discriminative validity studies. Good intraobserver reliability values were obtained for both researchers in Maastricht (MB.: weighted $\kappa=0.95$; C.G.F.: 0.90), with good interobserver scores as well (0.94). There was also a good interobserver reliability between the examiners at the 3 institutes: Maastricht-Milan: weighted $\kappa=0.78$; Maastricht-Ferrara: 0.83; Milan-Ferrara: 0.91.

In the subgroup of patients with sarcoidosis and possible SFN symptoms ( $\mathrm{n}=58), 19$ patients $(32.8 \%)$ had impaired IENFD scores. In contrast, all 


\begin{tabular}{|c|c|c|c|}
\hline \multirow[t]{2}{*}{ Table 1} & with or without sm & \multirow[b]{2}{*}{$\begin{array}{l}\text { Sarcoidosis } \\
\text { patients with } \\
\text { SFN symptoms }\end{array}$} & \multirow[b]{2}{*}{$\begin{array}{l}\text { Sarcoidosis } \\
\text { patients without } \\
\text { SFN symptoms }\end{array}$} \\
\hline & $\begin{array}{l}\text { Healthy } \\
\text { controls }\end{array}$ & & \\
\hline $\mathrm{n}$ & 188 & 58 & 14 \\
\hline \multicolumn{4}{|l|}{ Sex, n (\%) } \\
\hline Female & $97(51.6)$ & 25 (43.1) & $7(50)$ \\
\hline Male & 91 (48.4) & 33 (56.9) & $7(50)$ \\
\hline Age, mean (SD), range, $y$ & 47.0 (15.8), 20-82 & $45.4(9.2), 29-70$ & $46.5(9.5), 27-65$ \\
\hline $\begin{array}{l}\text { Duration of symptoms, } \\
\text { mean (SD), range, y }\end{array}$ & - & $7.9(6.0), 1-32$ & 9.4 (7.3), 2-28 \\
\hline \multicolumn{4}{|l|}{$\begin{array}{l}\text { Main initial reported } \\
\text { symptom", \% }\end{array}$} \\
\hline Burning feet & - & 1.7 & - \\
\hline Pain $^{+}$ & - & 27.6 & 28.6 \\
\hline Pulmonary ${ }^{\ddagger}$ & - & 32.8 & 28.6 \\
\hline Fatigue & - & 6.9 & 7.1 \\
\hline \multicolumn{4}{|l|}{$\begin{array}{l}\text { Main current reported } \\
\text { symptoms", \% }\end{array}$} \\
\hline Burning feet & - & 29.3 & - \\
\hline Pain $^{+}$ & - & 53.4 & 28.5 \\
\hline Pulmonary ${ }^{\ddagger}$ & - & 6.9 & 42.9 \\
\hline Fatigue & - & 96.6 & 85.7 \\
\hline
\end{tabular}

*Does not add up to $100 \%$ because of not separately mentioned symptoms (skin abnormalities, different autonomic symptoms, uveitis, fever, and weight loss).

'Various pain modalities were addressed: thoracic pain, joint pain, other pain in extremities, such as muscle pain.

*Dyspnea and cough were mentioned as the main pulmonary symptoms.

$\mathrm{SFN}=$ small fiber neuropathy

patients without SFN symptoms except 2 (14.3\%) had normal IENFD values ( $\chi^{2}$ test: $\left.p=0.17\right)$. For further discriminative validity studies, we divided the patients with SFN symptoms into those with IENFD being normal and those with IENFD $<5$ th percentile lower limit, ultimately having 3 subgroups for comparison (subgroup A: sarcoidosis without SFN symptoms, $\mathrm{n}=14$; subgroup B: sarcoidosis with SFN symptoms, normal IENFD findings, $\mathrm{n}=$ 39; subgroup C: sarcoidosis with SFN symptoms and abnormal IENFD values, $\mathrm{n}=19$ ). Figure 2 illustrates that more SFN-related symptoms as reported by SIQ are present in patients with abnormal IENFD, with a gradual transition between the 3 subgroups ( $\chi^{2}$ test between subgroups: $\left.p<0.001\right)$. Hypersensitivity is reported by $14 \%$ in subgroup $\mathrm{A}$, by $69 \%$ in subgroup B, and by $79 \%$ in subgroup C. Similar differences for symptoms of dry mouth $(50 \%, 72 \%, 79 \%)$ and orthostatic intolerance $(36 \%$, $67 \%, 84 \%)$ were found. Presence of SFN symptoms in subgroup A was comparable to that in healthy controls (data not shown). The SIQ demonstrated good test-retest reliability (weighted $\kappa=0.88$ ).

A significant discriminative validity was obtained when comparing the results of the PNQoL-97 domains between the 3 subgroups for pain, balance, energy/fatigue, upper extremity function, cognitive functioning, and social functioning. Also, the physical component score of this scale (V-PCS) showed a discriminative trend between the 3 subgroups of patients (table 3). In general, these PNQoL-97 domains and V-PCS did not significantly differentiate between subgroups B and C, patients with SFN symptoms, but showed a trend of more reduced quality-of-life features in those with abnormal IENFD (table 3). The remaining domains and

Figure 1 IENFD of 188 healthy individuals
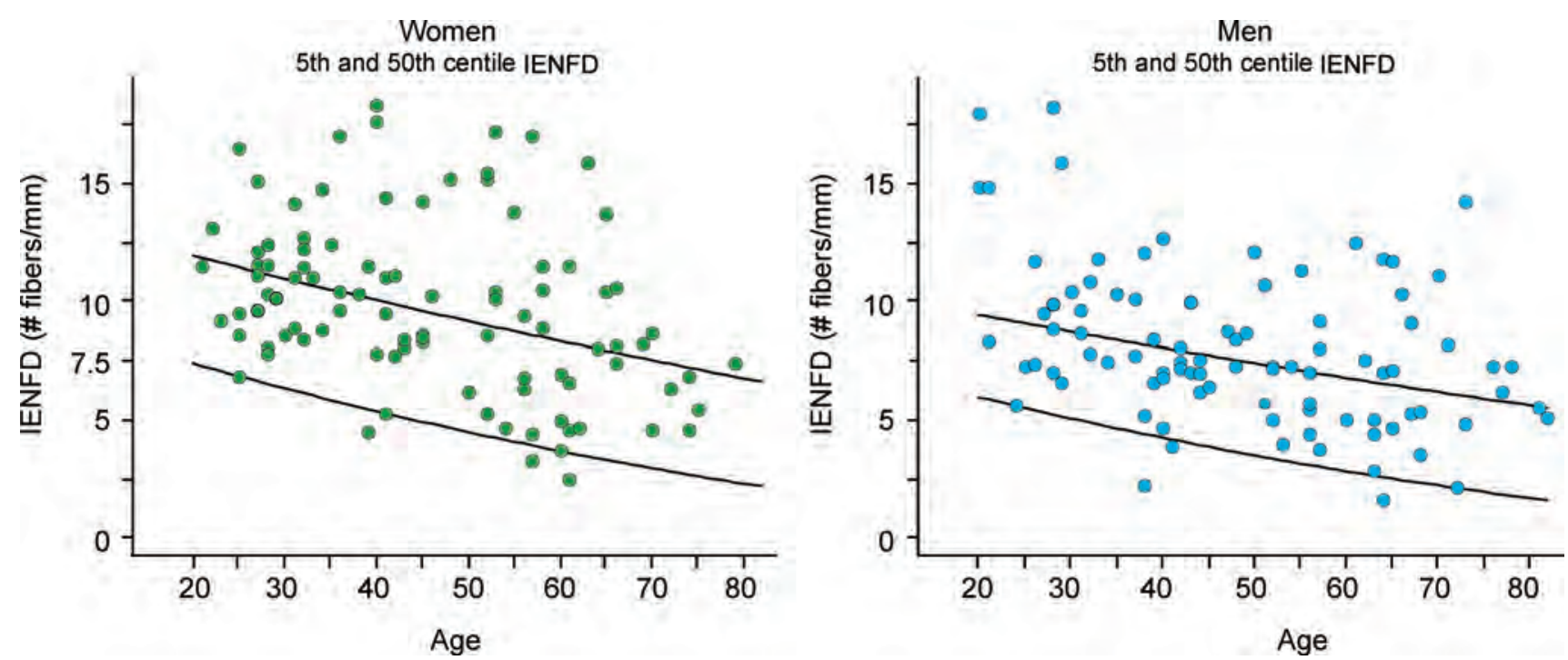

Scatterplot showing normative intraepidermal nerve fiber density (IENFD) scores in healthy individuals stratified for age and sex (5th and 50th percentiles shown). 


\begin{tabular}{|c|c|c|c|c|}
\hline Table 2 & Intraepiderma & rve fiber dens & normative values & clinical use \\
\hline \multirow[b]{2}{*}{ Age, y } & \multicolumn{2}{|l|}{ Women $(n=97)$} & \multicolumn{2}{|l|}{$\operatorname{Men}(n=91)$} \\
\hline & $\begin{array}{l}0.05 \text { quantile } \\
\text { IENFD values per } \\
\text { age span }\end{array}$ & $\begin{array}{l}\text { Median IENFD } \\
\text { values per } \\
\text { age span }\end{array}$ & $\begin{array}{l}0.05 \text { quantile } \\
\text { IENFD values per } \\
\text { age span }\end{array}$ & $\begin{array}{l}\text { Median IENFD } \\
\text { values per } \\
\text { age span }\end{array}$ \\
\hline $20-29$ & 6.7 & 11.2 & 5.4 & 9.0 \\
\hline $30-39$ & 6.1 & 10.7 & 4.7 & 8.4 \\
\hline $40-49$ & 5.2 & 9.9 & 4.0 & 7.8 \\
\hline $50-59$ & 4.1 & 8.7 & 3.2 & 7.1 \\
\hline $60-69$ & 3.3 & 7.9 & 2.4 & 6.3 \\
\hline$\geq 70$ & 2.7 & 7.2 & 2.0 & 5.9 \\
\hline
\end{tabular}

IENFD = intraepidermal nerve fiber density.

V-MCS did not show any difference between the subgroups of patients.

DISCUSSION In the current study, we described the determination of the largest series of normative distal leg IENFD values, stratified for age and sex. We demonstrated good interobserver/intraobserver reliability and acceptable IENFD validity scores through association with questionnaires. This series of skin biopsies is available for other researchers interested in joining a quality control program and in enlarging their normative reference values. Normative data for IENFD have been reported previously for the same location. ${ }^{20,22-24}$ Our results differ (lower values) from previous reports on IENFD normative values, which is most probably explained by differences in sample size of healthy controls and in count- ing rules. We found lower IENFD values in men compared with women, and an age-related decrease in IENFD, which is in conformity with these earlier reports.

Several neurologic tests have shown decreased function in healthy elderly, such as diminished grip strength, temperature and vibration sensation, and nerve conduction studies. ${ }^{25-28}$ Also, women have been reported to be more sensitive in quantitative sensory testing. ${ }^{29,30}$ On the structural level, agerelated decline is noted too, for example, in Meissner and Pacinian corpuscles. ${ }^{31,32}$ The reasons for agerelated decline and sex differences may be related to differences in hormonal status. ${ }^{33}$ Progesterone and its metabolites, for example, stimulate axonal growth and myelinization. Though it is also synthesized in the nervous system, in female rats, it is more abundant because of the gonadal production, and in experimental studies, nerve sprouting, regeneration, and remyelinization are more rapid and more complete in female animals. ${ }^{34,35}$

In our series, we were able to identify one-third of sarcoidosis patients with SFN using only sex- and age-related cutoff IENFD normative values. Our IENFD sensitivity turned out to be lower compared with reported literature findings, which is most probably related to differences in population of patients examined and differences in IENFD cutoff values. $^{36-38}$ We only included patients with pure small fiber-related symptoms and excluded patients with large fiber neuropathy. Others have examined

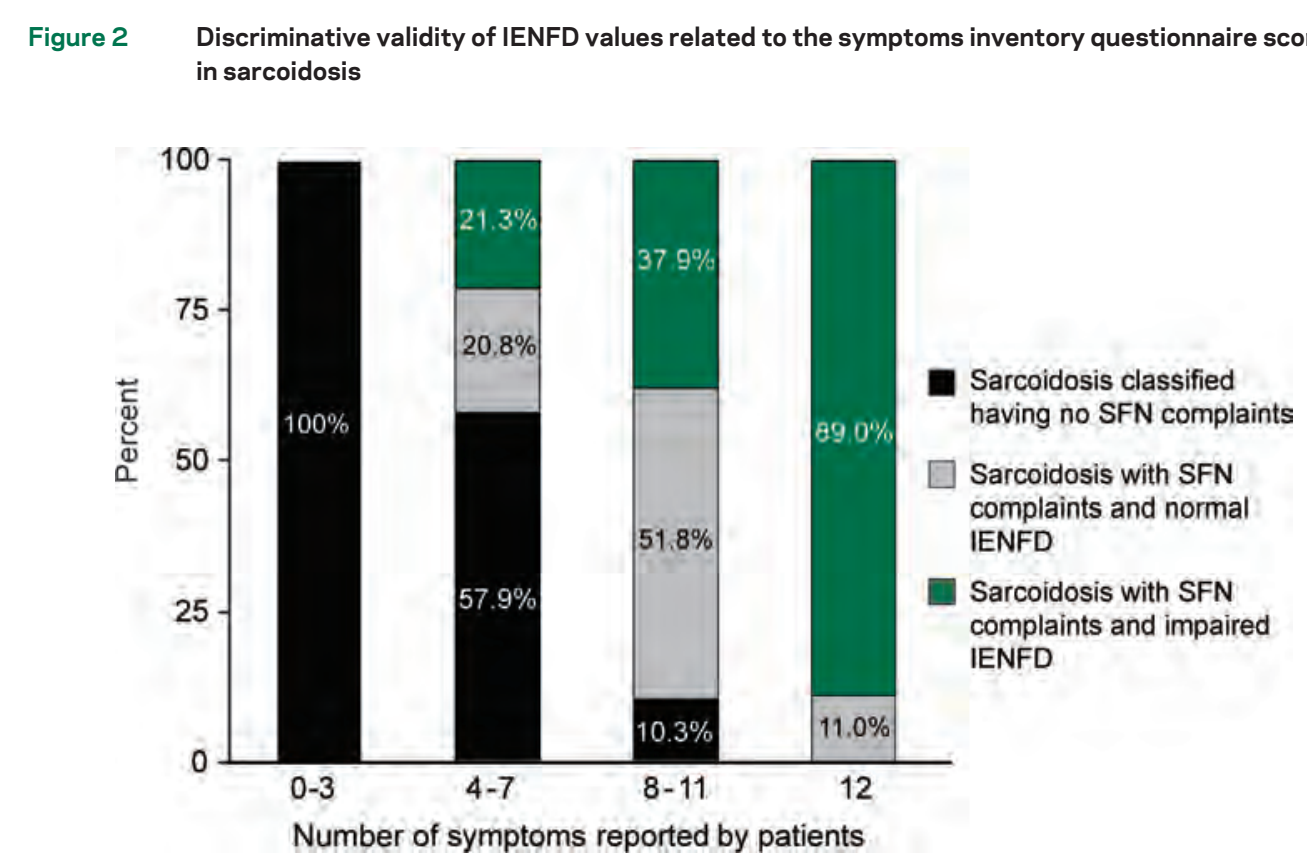

Bar chart showing patients with few (0-3), some (4-7), a lot (8-11), and all 12 symptoms, divided per subgroup: subgroup A, black $(n=14)$ : patients classified as having no small fiber neuropathy (SFN) symptoms; subgroup $B$, white $(n=39)$ : patients classified as having SFN symptoms, but normal intraepidermal nerve fiber density (IENFD) at examination; subgroup C, gray ( $n=19)$ : patients classified as having SFN symptoms and abnormal IENFD. 


\begin{tabular}{|lllll|}
\hline Table 3 & $\begin{array}{l}\text { Comparison of PNQoL-97 domain scores between subgroups of } \\
\text { patients with sarcoidosis }\end{array}$ & & \\
\hline Domain & $\begin{array}{l}\text { Subgroup } \\
\text { comparison } \\
<0.0001\end{array}$ & A vs B $^{+}$ & A vs C $^{+}$ & B vs C $^{+}$ \\
Pain & $<0.0001$ & $<0.0001$ & 0.057 \\
Balance & $<0.0001$ & 0.003 & $<0.0001$ & NS \\
$\begin{array}{l}\text { Energy/fatigue } \\
\text { Upper extremities }\end{array}$ & 0.01 & $\mathrm{NS}$ & 0.02 & $\mathrm{NS}$ \\
function & 0.004 & 0.01 & 0.005 & $\mathrm{NS}$ \\
$\begin{array}{l}\text { Cognitive functioning } \\
\text { Social functioning }\end{array}$ & 0.04 & 0.01 & $\mathrm{NS}$ & $\mathrm{NS}$ \\
$\begin{array}{l}\text { Physical component } \\
\text { score }\end{array}$ & 0.04 & 0.03 & $\mathrm{NS}$ & $\mathrm{NS}$ \\
\hline
\end{tabular}

Data are $p$ values.

*Overall result from analysis of variance with corrections according to Bonferroni multiple comparison tests.

+Subgroup A: patients without small fiber neuropathy (SFN)-related symptoms; subgroup B: patients with SFN-related symptoms and normal intraepidermal nerve fiber density (IENFD); subgroup C: patients with SFN-related symptoms and reduced IENFD.

PNQoL-97 = Vickrey Peripheral Neuropathy Quality-of-Life Instrument-97; NS = not significant.
However, the validation of this proposition is beyond the scope of the current study. Second, some caution is required when interpreting the results, because the sample sizes of the various subgroups were relatively small. Third, efforts were taken to subdivide the 72 selected patients into 3 subgroups based on a systematic interview focusing on the presence of SFN symptoms. Despite these efforts, patients of the subgroup without any SFN symptoms (subgroup A) reported through the SIQ questionnaire some presence of symptoms, although relatively low compared with the other 2 subgroups of patients (figure 2). However, the presence of symptoms in subgroup A was similar to that in healthy controls. Finally, we have not systematically examined whether skin type or occupation might have any influence on IENFD.

The current study presents clinically applicable distal leg IENFD values, supporting previously reported age- and sex-related differences. Validity through association with an SFN-specific and generic quality-of-life questionnaire was acceptable. Hence, IENFD is suggested as an alternative endpoint in future clinical trials in neuropathies, as has been addressed previously. ${ }^{40}$ Further study in patients with SFN and validation of a possible gold standard are recommended.

these patients compared with those with only sn nerve fiber involvement. ${ }^{37,38}$ Progression from pure SFN to large fiber involvement in patients over time with further reduction of IENFD in these patients has also been reported, hence underlining the notion of a continuum and progressive disease pattern. ${ }^{36}$

The current article addresses discriminatory validity between subgroups of patients with sarcoidosis, translating pathology (small fiber findings in skin biopsy) to impairments (SFN symptoms) and partly to quality-of-life expectations, by demonstrating differences between sarcoidosis patients without SFN symptoms vs those with SFN symptoms but normal IENFD vs those with SFN symptoms and impaired IENFD. Similar findings have been reported in patients with various forms of inflammatory neuropathies. ${ }^{39}$ Although not significant, patients with SFN symptoms and impaired IENFD had more physically oriented symptoms, such as more pain, less energy, and more balance problems, and more social dysfunction compared with the other subgroups. Emotional items scores did not differ between subgroups, thus excluding emotional problems as an explanation for patients' dysfunction. Hence, low IENFD contributes to the patients' daily symptoms and physical burden and helps clinicians in understanding its impact in patients with sarcoidosis.

There are some methodologic issues that should be addressed. First, a gold standard for the diagnosis SFN was proposed: the presence of at least 2 abnormal results at clinical, QST, and skin biopsy examinations. ${ }^{36}$

\section{AUTHOR CONTRIBUTIONS}

Statistical analysis was performed by Ingemar Merkies, MD, $\mathrm{PhD}$.

\section{ACKNOWLEDGMENT}

The authors thank Prof. M. Drent (Department of Pulmonology, head of the Interstitial Lung Disease Center) for patient referral. Prof. M. van Kleef (Department of Anesthesiology, MUMC) provided the authors with useful comments on the manuscript. The authors also thank J. Smeets (Department of Pathology, MUMC) and F. Camozzi (Carlo Besta National Neurological Institute) for their work and support in the laboratory.

\section{DISCLOSURE}

Dr. Bakkers received an honorarium from Profileringsfonds [062053]. Dr. Merkies served on a Steering Committee for and received research support from Talecris Biotherapeutics Co.; and received research support from the GBS/CIDP International Foundation. Dr. Lauria served on scientific advisory boards for Medtronic, Mitsubishi, and GlaxoSmithKline; has received funding for travel from Pzifer, Inc. and Kedrion; serves on the editorial board of the Journal of the Peripheral Nervous System; has received speaker honoraria from Boehringer and Formenti-Grunenthal; and has received research support from Formenti-Grunenthal, Pfizer, Inc., Agave, and the Italian Ministry of Health [RF-2005/51]. Dr. Devigili received research support from BERCO S.p.A., Copparo, and Fondazione Cassa di Risparmio di Ferrara. Dr. Hermans received an honorarium from the Prinses Beatrix Fonds. Prof. de Baets has received research support from the Prinses Beatrix Fonds, the Transnational University Limburg, Genmab, and the European Union [30973317E, 2005105, and LSHM-CT-2006037833]. Dr. Faber received research support from the Prinses Beatrix Fonds and from the Profileringsfonds MUMC. Dr. Penza, Dr. Lombardi, and Dr. van Nes report no disclosures.

Received December 30, 2008. Accepted in final form July 16, 2009. 


\section{REFERENCES}

1. Mendell JR, Sahenk Z. Clinical practice. Painful sensory neuropathy N Engl J Med 2003;348:1243-1255.

2. Lauria G. Small fibre neuropathies. Curr Opin Neurol 2005;18:591-597.

3. Lacomis D. Small-fiber neuropathy. Muscle Nerve 2002; 26:173-188.

4. Hoitsma E, Reulen JP, de Baets M, et al. Small fiber neuropathy: a common and important clinical disorder. J Neurol Sci 2004;227:119-130.

5. Lauria G, Lombardi R. Skin biopsy: a new tool for diagnosing peripheral neuropathy. BMJ 2007;334:1159-1162.

6. Hoitsma E, Marziniak M, Faber CG, et al. Small fibre neuropathy in sarcoidosis. Lancet 2002;359:2085-2086.

7. Lauria G, Cornblath DR, Johansson O, et al. EFNS guidelines on the use of skin biopsy in the diagnosis of peripheral neuropathy. Eur J Neurol 2005;12:747-758.

8. Sommer C, Lauria G. Skin biopsy in the management of peripheral neuropathy. Lancet Neurol 2007;6:632-642.

9. Ebenezer GJ, Hauer P, Gibbons C, McArthur JC, Polydefkis M. Assessment of epidermal nerve fibers: a new diagnostic and predictive tool for peripheral neuropathies. J Neuropathol Exp Neurol 2007;66:1059-1073.

10. Smith AG, Howard JR, Kroll R, et al. The reliability of skin biopsy with measurement of intraepidermal nerve fiber density. J Neurol Sci 2005;228:65-69.

11. Hunninghake GW, Costabel U, Ando M, et al. ATS/ERS/ WASOG statement on sarcoidosis. American Thoracic Society/European Respiratory Society/World Association of Sarcoidosis and Other Granulomatous Disorders Sarcoidosis. Vasc Diffuse Lung Dis 1999;16:149-173.

12. Lauria G, Penza P, Melli G, et al. European interlaboratory agreement project on skin biopsy standardization and innervation count. J Peripher Nerv Syst 2007;12: 49-50. Abstract.

13. Hoitsma E. Small Fiber Neuropathy: A Novel Finding in Sarcoidosis. Maastricht: University of Maastricht; 2005.

14. Streiner DL, Norman GR. Health Measurement Scales: A Practical Guide to Their Development and Use. 2nd ed. New York: Oxford University Press; 1998.

15. Ware JE Jr, Snow KK, Kosinski M, Gandek B. SF-36 Health Survey: Manual and Interpretation Guide. Boston: The Health Institute, New England Medical Center; 1993.

16. Vickrey BG, Hays RD, Beckstrand M. Development of a health-related quality of life measure for peripheral neuropathy. Neurorehabil Neural Repair 2000;14:93-104.

17. Ware JE Jr, Gandek B, Kosinski M, et al. The equivalence of SF-36 summary health scores estimated using standard and country-specific algorithms in 10 countries: results from the IQOLA Project. International Quality of Life Assessment. J Clin Epidemiol 1998;51:1167-1170.

18. Ware JE Jr, Kosinski M, Keller SD. SF-36 Physical and Mental Health Summary Scales: A User's Manual. Boston: The Health Assessment Lab, New England Medical Center; 1994.

19. Jorgensen CK, Karlsmose B. Validation of automated forms processing: a comparison of Teleform with manual data entry. Comput Biol Med 1998;28:659-667.

20. McArthur JC, Stocks EA, Hauer P, Cornblath DR, Griffin JW. Epidermal nerve fiber density: normative reference range and diagnostic efficiency. Arch Neurol 1998;55: 1513-1520.
21. Cohen J. Weighted kappa: nominal scale agreement with provision for scale disagreement or part credit. Psychol Bull 1968;70:213-230.

22. Chang YC, Lin WM, Hsieh ST. Effects of aging on human skin innervation. Neuroreport 2004;15:149-153.

23. Goransson LG, Mellgren SI, Lindal S, Omdal R. The effect of age and gender on epidermal nerve fiber density. Neurology 2004;62:774-777.

24. Umapathi T, Tan WL, Tan NC, Chan YH. Determinants of epidermal nerve fiber density in normal individuals. Muscle Nerve 2006;33:742-746.

25. Martina IS, van Koningsveld R, Schmitz PI, van der Meche FG, van Doorn PA. Measuring vibration threshold with a graduated tuning fork in normal aging and in patients with polyneuropathy. European Inflammatory Neuropathy Cause and Treatment (INCAT) group. J Neurol Neurosurg Psychiatry 1998;65:743-747.

26. Merkies IS, Schmitz PI, Samijn JP, et al. Assessing grip strength in healthy individuals and patients with immunemediated polyneuropathies. Muscle Nerve 2000;23:1393-1401.

27. Lin YH, Hsieh SC, Chao CC, Chang YC, Hsieh ST. Influence of aging on thermal and vibratory thresholds of quantitative sensory testing. J Peripher Nerv Syst 2005;10: 269-281.

28. Rivner MH, Swift TR, Malik K. Influence of age and height on nerve conduction. Muscle Nerve 2001; 24:1134-1141.

29. Meh D, Denislic M. Quantitative assessment of thermal and pain sensitivity. J Neurol Sci 1994;127:164-169.

30. Jain S, Muzzafarullah S, Peri S, et al. Lower touch sensibility in the extremities of healthy Indians: further deterioration with age. J Peripher Nerv Syst 2008;13:47-53.

31. Bolton CF, Winkelmann RK, Dyck PJ. A quantitative study of Meissner's corpuscles in man. Neurology 1966; 16:1-9.

32. Bruce MF, Sinclair DC. The relationship between tactile thresholds and histology in the human finger. J Neurol Neurosurg Psychiatry 1980;43:235-242.

33. Roglio I, Giatti S, Pesaresi M, et al. Neuroactive steroids and peripheral neuropathy. Brain Res Rev 2008;57:460-469.

34. Koenig HL, Gong WH, Pelissier P. Role of progesterone in peripheral nerve repair. Rev Reprod 2000;5:189-199.

35. Kovacic U, Sketelj J, Bajrovic FF. Sex-related difference in collateral sprouting of nociceptive axons after peripheral nerve injury in the rat. Exp Neurol 2003;184:479-488.

36. Devigili G, Tugnoli V, Penza P, et al. The diagnostic criteria for small fibre neuropathy: from symptoms to neuropathology. Brain 2008;131:1912-1925.

37. Scherens A, Maier C, Haussleiter IS, et al. Painful or painless lower limb dysesthesias are highly predictive of peripheral neuropathy: comparison of different diagnostic modalities. Eur J Pain 2009;13:711-718.

38. Obermann M, Katsarava Z, Esser S, et al. Correlation of epidermal nerve fiber density with pain-related evoked potentials in HIV neuropathy. Pain 2008;138:79-86.

39. Merkies IS, Schmitz PI, van der Meche FG, Samijn JP, van Doorn PA. Connecting impairment, disability, and handicap in immune mediated polyneuropathies. J Neurol Neurosurg Psychiatry 2003;74:99-104.

40. Merkies IS, Lauria G. 131st ENMC International Workshop: selection of outcome measures for peripheral neuropathy clinical trials 10-12 December 2004, Naarden, The Netherlands. Neuromuscul Disord 2006;16:149-156. 